\title{
A Phase II Trial of Preoperative Chemoradiotherapy with Oral DPD-Inhibitory Fluoropyrimidines in Patients with Advanced Rectal Cancer ${ }^{*}$
}

\author{
Masanori Hotchi $^{1 \#}$, Hiroshi Okitsu ${ }^{2}$, Murato Miura ${ }^{3}$, Madoka Hamada ${ }^{4}$, Yasuhide Sonaka ${ }^{5}$, \\ Yoh Fukuda ${ }^{6}$, Tsutomu Ando ${ }^{7}$, Shinji Kuratate ${ }^{8}$
}

${ }^{1}$ Department of Surgery, Tokushima University Hospital, Tokushima, Japan; ${ }^{2}$ Department of Surgery, Tokushima Red Cross Hospital, Tokushima, Japan; ${ }^{3}$ Department of Surgery, Shikoku Central Hospital, Ehime, Japan; ${ }^{4}$ Department of Surgery, Kochi Health Sciences Center, Kochi, Japan; ${ }^{5}$ Department of Surgery, Tokushima Municipal Hospital, Tokushima, Japan; ${ }^{6}$ Department of Surgery, Takamatsu City Hospital, Takamatsu, Japan; ${ }^{7}$ Department of Surgery, National Hospital Organization Kochi National Hospital, Kochi, Japan; ${ }^{8}$ Department of Surgery, Tokushima Prefectural Central Hospital, Tokushima, Japan.

Email: "mn1029keiko0524@yahoo.co.jp

Received August $9^{\text {th }}, 2012$; revised September $10^{\text {th }}, 2012$; accepted September $19^{\text {th }}, 2012$

\begin{abstract}
Background: Preoperative chemoradiotherapy (CRT) with 5-FU has been widely used to improve local control of disease and to preserve anal sphincter in the treatment of rectal cancer. UFT and S-1 as oral dihydropyrimidine dehydrogenase (DPD)-inhibitory fluoropyrimidines enhance the therapeutic effect of 5-FU by modulating its metabolic pathways. The purpose of this study was to evaluate the efficacy and toxicity of CRT using UFT versus S-1 in patients with locally advanced rectal cancer. Methods: Fifty-nine patients who received preoperative CRT (40 Gy radiotherapy) were randomly assigned to either UFT or S-1 groups. UFT and S-1 were administered during the radiotherapy course. Response to CRT was determined using a histopathologic examination and RECIST of surgically resected specimens and classified as responders (CR, PR and Grade 2, 3) or nonresponders (SD, PD and Grade 0,1). Results: All patients were randomly allocated to S-1 group $(n=30)$ or UFT group $(n=29)$. Pathological response rate (Grade 2 and Grade 3$)$ was $57 \%$ in the S-1 group and $45 \%$ in the UFT group $(\mathrm{p}=0.36)$. Pathological complete response (CR) rate (Grade 3$)$ was $7 \%$ in the S-1 group and $4 \%$ in the UFT group $(\mathrm{p}=0.98)$. There was no statistically significant difference between the two groups in regard to the response rate of RECIST $(p=0.52)$. There was no statistically significant difference between the groups based on the downstaging rate, resection of tumor, sphincter preservation and marginal invasion. The incidence of Grade 3 diarrhea was significantly more frequent in the S-1 group (7\%) compared with the UFT group (0\%) $(\mathrm{p}=$ 0.02). Conclusion: The results supported the conclusion that CRT using UFT or S-1 is effective and feasible for patients with locally advanced rectal cancer.
\end{abstract}

Keywords: S-1; UFT; Chemoradiation; Rectal Cancer

\section{Introduction}

Advanced rectal cancer carries a poorer prognosis than advanced colon cancer. Locoregional recurrence after resection of rectal cancer is difficult to treat and is associated with severe debilitating symptoms. Meta-analyses have proven that preoperative radiotherapy compared with surgery alone significantly increases locoregional control and survival [1,2]. On the other hand, a large Phase III study showed that the control of local recurrence at 5

*Drs. Masanori Hotchi, Hiroshi Okitsu, Murato Miura, Madoka Hamada, Yasuhide Sonaka, Yoh Fukuda, Tsutomu Ando and Shinji Kuratate have no conflicts of interest or financial ties to disclose.

${ }^{\#}$ Corresponding author. years was significantly better in patients who received chemoradiotherapy (CRT) than in those who received radiotherapy alone [3]. In response to these outcomes, preoperative CRT has been widely used to improve local control of disease and to preserve anal sphincter in the treatment of locally advanced rectal cancer.

5-Fluorouracul (5-FU) is a chemotherapeutic agent widely used for CRT of locally advanced rectal cancer. 5-FU is phosphorylated into active metabolites and kills tumor cells mainly through inhibition of thymidylate synthase (TS), which is a key enzyme in de novo DNA synthesis. 5-FU is also degraded by dihydropyrimidine dehydrogenase (DPD), and high DPD activity that promotes 5-FU degradation as well as high TS activity that 
cannot be inhibited by 5 -FU may cause reduced antitumor effect of 5-FU. Thus, to enhance the therapeutic effect of 5-FU by modulating its metabolic pathways, a number of 5-FU derivative agents have been developed. Among them, UFT and S-1, defined as oral DPD-inhibitory fluoropyrimidines (DIFs). UFT and S-1 are oral agents in which a pro-drug of 5-FU, tegafur (FT), is combined with a DPD inhibitor; as a DPD-inhibitor, uracil is used in UFT [4] and 5-chloro-2,4-dihydroxypyridine (CDHP, gimeracil) is used in S-1 [5,6]. 5-FU is gradually released from FT, and a certain 5-FU concentration can be maintained for a long period as degradation of 5-FU is inhibited by a DPD inhibitor. Thus, UFT and $\mathrm{S}-1$ are defined as oral DIFs and have the clinical advantage of having an enhanced antitumor effect achieved by a prolonged intratumoral 5-FU concentration as well as the administration convenience owing to its oral formula. Additionally, in S-1, potassium oxonate (Oxo) is also combined to prevent GI toxicity without loss of the antitumor effect, as Oxo inhibits phosphorylation of 5FU in the GI tract but not in tumor tissues [6].

Phase II studies have shown that the UFT/radiotherapy combination is effective and well tolerated in the preoperative treatment of rectal cancer [7-11]. On the other hand, CDHP (gimeracil), a component of S-1, has potent radiosensitizing property [12]. Additionally, as CDHP shows a 180 times higher DPD-inhibitory effect compared with uracil, S-1 may become an active drug for CRT of locally advanced rectal cancer. We previously performed a Phase I clinical study and determined the recommended dose $\left(80 \mathrm{mg} / \mathrm{m}^{2}\right)$ of S-1 for preoperative CRT [13]. In the Phase I study, the dose-limiting toxicity was diarrhea. The purpose of this study was to evaluate the efficacy and toxicity of preoperative CRT using UFT versus S-1 in patients with locally advanced rectal cancer and as the results indicate the outcome of the study provided positive treatment options with CRT using UFT or S-1.

\section{Patients and Methods}

\subsection{Patients}

A total of 60 patients were required with a power of $90 \%$ and a $5 \%$ single-sided significance level for detection of a $25 \%$ increase in response rate in the combined experimental arm. From April 2008 to October 2010, 62 patients with locally advanced rectal cancer within $10 \mathrm{~cm}$ from the anal verge were enrolled from multi-institutions. Patients were randomly assigned to receive preoperative CRT with S-1 (S-1 group) or UFT (UFT group). Random allocation of participants to S-1 group or UFT group was performed by a person not involved in the care or evalua- tion of the patients. Although three patients signed the consent form, they refused preoperative CRT before treatment and their operations were performed operation without CRT. So, 59 patients were analyzed and fulfilled the following criteria before treatment: Eastern Cooperative Oncology Group (ECOG) performance status of 0 to 2 , normal bone marrow function (white blood count $\geq$ $4000 / \mathrm{mm}^{3}$, platelet count $\geq 100,000 / \mathrm{mm}^{3}$ ), liver function (serum total bilirubin $<1.5 \mathrm{mg} / \mathrm{dl}$ ), renal function (creatinine $<1.5 \mathrm{mg} / \mathrm{dl}$ ), and heart function (stable cardiac rhythm, no active angina, no clinical evidence of congestive heart failure). Evaluation of patients included physical examination, digital rectal examination, colonoscopy with biopsy, pelvic magnetic resonance imaging (MRI), abdominal and pelvic contrast computed tomography (CT) scan and positron emission tomography/CT scan (PET/CT). Rigid rectoscopy was performed to assess the exact level of the tumor from the anal verge. Informed consent was obtained from all patients included in the study, which was approved by local ethics committees. This study was registered in UMIN (000001704).

\subsection{Preoperative CRT}

At our institution, CRT is routinely offered to patients with locally advanced ( $\geq \mathrm{T} 3$ and/or node positive) cancers at the time of diagnosis or to those who have very distal T2N0 cancers close to or involving the sphincter. All patients received CRT with a total dose of 40 Gy of pelvic irradiation, which was administered five times weekly, with a daily fraction of 2 Gy utilizing a four-field technique. The top of the radiation field consisted of the bifurcation point of the aorta, and the bottom of the field was marked at least $4 \mathrm{~cm}$ below the tumor. Radiation was delivered concomitantly with S-1 or UFT. The S-1 (80 $\left.\mathrm{mg} / \mathrm{m}^{2}\right)$ or UFT $\left(300 \mathrm{mg} / \mathrm{m}^{2}\right)$ was administered on days of radiation. If patients had Grade 3 toxicity, the S-1 or UFT dose was reduced to $75 \%$ of the previous dose. Chemotherapy was delayed until recovery if the neutrophil count decreased to less than $1500 / \mathrm{L}$ or the platelet count decreased to less than $100,000 / \mathrm{L}$. Surgical treatment was performed 6 - 8 weeks after completion of preoperative CRT. Repeat examination was performed for post-neoadjuvant CRT evaluation. The design of preoperative CRT is shown in Figure 1.

\subsection{Evaluation of Efficacy and Toxicity of CRT}

The primary end point of this study was the pathological response rate. The secondary end points were the response rate (RECIST), pathological complete response (CR) rate and frequency of adverse effects. Histopathologic examinations of surgically resected specimens were based on a semiquantitative classification system as 


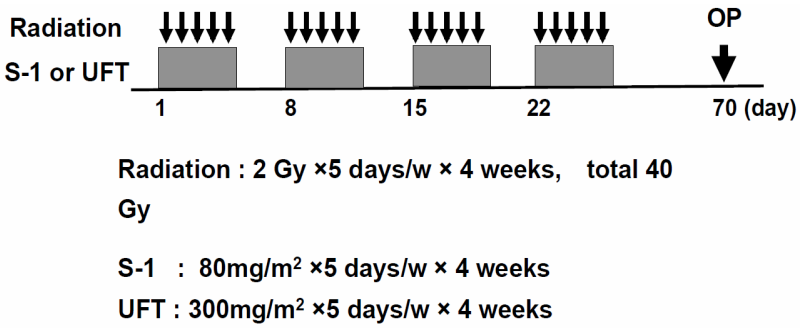

Figure 1. Design of preoperative CRT. 2 Gy strong radiation was done for 5 days in a week, for 4 weeks, and S-1 (80 $\left.\mathrm{mg} / \mathrm{m}^{2}\right)$ or UFT $\left(300 \mathrm{mg} / \mathrm{m}^{2}\right)$ was administered orally on same day. Radiation therapy was used four field box technique.

described in detail previously [14]. Tumors were classified as responder when assigned to the regression Grade 2 or 3, and non-responder when Grade 0 or 1 . Additionally, the anti-tumor effect of CRT was assessed by the Guidelines for Evaluation of the Response to Treatment in Solid Tumors (RECIST). Adverse effects of preoperative CRT were assessed using the National Cancer Institute's (NCI) common toxicity criteria (CTC).

\subsection{Statistical Analysis}

Quantitative data were given as median (range). Comparisons of clinicopathological and surgical data were performed using a Fisher's exact test, chi-square test and Mann-Whitney U test, as appropriate. All statistical tests performed were two-sided and declared at the 5\% significance level. All statistical analysis was performed using statistical software (JMP 8.0.1., SAS Campus Drive, Cary, 27513 NC, USA).

\section{Results}

\subsection{Patient Characteristics}

All patients were randomly allocated to $\mathrm{S}-1$ group $(\mathrm{n}=$ $30)$ or UFT group $(n=29)$. The population in the $S-1$ group consisted of 17 men and 13 women with a median age of 67 years. The population in the UFT group consisted of 6 men and 23 women with a median age of 64 years. The majority of PS in both groups were frequently PS 0 and 1. Median tumor size and tumor distance from the anal verge were same in the two groups. The majority of tumors in both groups were frequently well and moderately differentiated. Tumor stage was similar in both groups as well. The population in the S-1 group consisted of $12(40 \%) \mathrm{N} 0,9(30 \%) \mathrm{N} 1$ and $9(30 \%) \mathrm{N} 2$ and 12 $(41 \%) \mathrm{N} 0,12(41 \%) \mathrm{N} 1$ and $5(18 \%) \mathrm{N} 2$ in the UFT group. Except for gender there was no statistically significant difference between the groups based on any of these parameters (Table 1).
Table 1. Comparison of patient characteristics.

\begin{tabular}{|c|c|c|c|}
\hline & $\mathrm{S}-1(\mathrm{n}=30)$ & UFT $(\mathrm{n}=29)$ & $\mathrm{p}$ value \\
\hline Gender & & & 0.01 \\
\hline Male & $17(57 \%)$ & $6(21 \%)$ & \\
\hline Female & $13(43 \%)$ & $23(79 \%)$ & \\
\hline Age (years) & $67(38-80)$ & $64(47-78)$ & 0.64 \\
\hline Performance status & & & 0.44 \\
\hline 0 & $27(90 \%)$ & $25(86 \%)$ & \\
\hline 1 & $2(7 \%)$ & $4(14 \%)$ & \\
\hline 2 & $1(3 \%)$ & $0(0 \%)$ & \\
\hline Tumor size (cm) & $4.0(2.5-9.0)$ & $4.0(2.0-12.0)$ & 1.00 \\
\hline $\begin{array}{c}\text { Tumor distance from the anal } \\
\text { verge }(\mathrm{cm})\end{array}$ & $3.0(0-9.0)$ & $3.0(1.0-9.0)$ & 0.95 \\
\hline Grade of differentiation & & & 0.99 \\
\hline Well/moderately & $29(97 \%)$ & $29(100 \%)$ & \\
\hline Poorly/mucinous & $1(3 \%)$ & $0(0 \%)$ & \\
\hline Tumor stage & & & 0.90 \\
\hline $\mathrm{T} 2$ & $3(10 \%)$ & $4(14 \%)$ & \\
\hline $\mathrm{T} 3$ & $23(77 \%)$ & $21(72 \%)$ & \\
\hline $\mathrm{T} 4$ & $4(13 \%)$ & $4(14 \%)$ & \\
\hline Nodal stage & & & 0.46 \\
\hline No & $12(40 \%)$ & $12(41 \%)$ & \\
\hline N1 & $9(30 \%)$ & $12(41 \%)$ & \\
\hline N2 & $9(30 \%)$ & $5(18 \%)$ & \\
\hline
\end{tabular}

\subsection{Efficacy of CRT}

Table 2 summarizes response of preoperative CRT. The population of pathological response Grade of CRT in the S-1 group consisted of $6(20 \%)$ Grade 1a, 7 (23\%) Grade 1b, $15(50 \%)$ Grade 2 and 2 (7\%) Grade 3 and $7(24 \%)$ Grade 1a, 9 (31\%) Grade 1b, 12 (41\%) Grade 2 and 1 (4\%) Grade 3 in the UFT group. Pathological response rate (Grade 2 and Grade 3) of the resection specimens was $57 \%$ in the S- 1 group and $45 \%$ in the UFT group $(\mathrm{p}=$ 0.36). Pathological CR rate (Grade 3) was 7\% in the S-1 group and $4 \%$ in the UFT group $(p=0.98)$. There was no statistically significant difference between the two groups in regard to the pathological response rate and pathological CR rate.

In regard to RECIST, although CR was observed in 1 patient (3\%) in the S-1 group, none was observed in the UFT group. A partial response was observed in 17 patients $(57 \%)$ in the S-1 group and in 15 patients $(52 \%)$ in the UFT group. A stable disease was observed in 12 patients (40\%) in the S-1 group and in 14 patients (48\%) in the UFT group. No progressive disease was observed in either group. There was no statistically significant difference between the two groups in regard to the response 
Table 2. Comparison of response to CRT.

\begin{tabular}{|c|c|c|c|}
\hline & $\mathrm{S}-1(\mathrm{n}=30)$ & UFT $(\mathrm{n}=29)$ & $\mathrm{p}$ value \\
\hline Pathological response & & & 0.81 \\
\hline Grade 1a & $6(20 \%)$ & $7(24 \%)$ & \\
\hline Grade $1 \mathrm{~b}$ & $7(23 \%)$ & $9(31 \%)$ & \\
\hline Grade 2 & $15(50 \%)$ & $12(41 \%)$ & \\
\hline Grade 3 & $2(7 \%)$ & $1(4 \%)$ & \\
\hline $\begin{array}{l}\text { Pathological response rate } \\
\qquad(\text { Grade } 2+3)\end{array}$ & $17(57 \%)$ & $13(45 \%)$ & 0.36 \\
\hline $\begin{array}{l}\text { Pathological CR rate } \\
\text { (Grade } 3 \text { ) }\end{array}$ & $2(7 \%)$ & $1(4 \%)$ & 0.98 \\
\hline RECIST & & & 0.71 \\
\hline Complete response & $1(3 \%)$ & $0(0 \%)$ & \\
\hline Partial response & $17(57 \%)$ & $15(52 \%)$ & \\
\hline Stable disease & $12(40 \%)$ & $14(48 \%)$ & \\
\hline Progressive disease & $0(0 \%)$ & $0(0 \%)$ & \\
\hline $\begin{array}{l}\text { Response rate } \\
(\mathrm{CR}+\mathrm{PR})\end{array}$ & $18(60 \%)$ & $15(52 \%)$ & 0.52 \\
\hline
\end{tabular}

rate (CR and partial response) $(\mathrm{p}=0.52)$.

The other tumor response to preoperative CRT is shown in Table 3. Downstaging rate was $47 \%$ in the S-1 group and $59 \%$ in the UFT group. Resection of tumor was performed in 29 patients (97\%) in the S-1 group and in 29 patients $(100 \%)$ in the UFT group. Anal sphincter was preserved in 14 patients (47\%) in the S-1 group and in 17 patients (59\%) in the UFT group. Downstaging rate was $47 \%$ in the S-1 group and $59 \%$ in the UFT group. Distal marginal invasion and radial marginal invasion were $0 \%$ and $3 \%$ in the S- 1 group and $0 \%$ and $0 \%$ in the UFT group, respectively. There was no statistically significant difference between the groups based on any of these parameters.

\subsection{Adverse Effects of CRT}

Adverse events as acute toxicities of CRT are summarized in Table 4. Adverse events are infrequent, and there was no Grade 4 hematologic or non-hematologic toxicity. Regarding hematologic toxicity, only 1 patient had Grade 3 anemia and 1 had Grade 3 thrombocytopenia in the S-1 group. No patient with Grade 3 toxicity was observed in UFT group. Regarding non-hematologic toxicity, Grade 3 nausea was observed in 1 patient and Grade 3 diarrhea was observed in 2 patients in the S-1 group. In S-1 group, three patients had several Grade 3 toxicities. The first patient with Grade 3 diarrhea concurrently had Grade 3 thrombocytopenia, which promptly improved after treatment with a continuous intravenous infusion. The second patient with Grade 3 nausea concurrently had Grade 3 anemia. And the third patient with Grade 3 diarrhea concurrently had Grade 3 fatigue and
Table 3. Comparison of produced effects by CRT.

\begin{tabular}{cccc}
\hline & S-1 $(\mathrm{n}=30)$ & UFT $(\mathrm{n}=29)$ & $\mathrm{p}$ value \\
\hline Down staging & & & 0.36 \\
Yes & $14(47 \%)$ & $17(59 \%)$ & \\
No & $16(53 \%)$ & $12(41 \%)$ & \\
Resection of tumor & & & 0.99 \\
Yes & $29(97 \%)$ & $29(100 \%)$ & \\
No & $1(3 \%)$ & $0(0 \%)$ & \\
Sphincter preserve & & & 0.36 \\
Yes & $14(47 \%)$ & $17(59 \%)$ & \\
No & $16(53 \%)$ & $12(41 \%)$ & \\
Marginal invasion & & & \\
Distal margin & $0(0 \%)$ & $0(0 \%)$ & \\
Radial margin & $1(3 \%)$ & $0(0 \%)$ & 0.99 \\
\hline
\end{tabular}

Table 4. Comparison of adverse events of CRT.

\begin{tabular}{cccccc}
\hline & \multicolumn{2}{c}{$\mathrm{S}-1(\mathrm{n}=30)$} & \multicolumn{2}{c}{ UFT $(\mathrm{n}=29)$} & $\mathrm{p}$ value \\
\hline Hematologic & $\mathrm{G} 2$ & $\mathrm{G} \mathrm{3}$ & $\mathrm{G} 2$ & $\mathrm{G} \mathrm{3}$ & \\
Anemia & $1(3 \%)$ & $1(3 \%)$ & $1(3 \%)$ & $0(0 \%)$ & 0.61 \\
Leukopenia & $4(13 \%)$ & $0(0 \%)$ & $3(10 \%)$ & $0(0 \%)$ & 0.93 \\
Thrombocytopenia & $0(0 \%)$ & $1(3 \%)$ & $0(0 \%)$ & $0(0 \%)$ & 0.58 \\
& & & & & \\
Non-hematologic & & & & & \\
Nausea & $0(0 \%)$ & $1(3 \%)$ & $0(0 \%)$ & $0(0 \%)$ & 0.58 \\
Diarrhea & $4(13 \%)$ & $2(7 \%)$ & $0(0 \%)$ & $0(0 \%)$ & 0.02 \\
Anorexia & $1(3 \%)$ & $0(0 \%)$ & $0(0 \%)$ & $0(0 \%)$ & 0.58 \\
Fatigue & $0(0 \%)$ & $1(3 \%)$ & $0(0 \%)$ & $0(0 \%)$ & 0.58 \\
Hyponatremia & $0(0 \%)$ & $1(3 \%)$ & $0(0 \%)$ & $0(0 \%)$ & 0.58 \\
Creatinine increased & $1(3 \%)$ & $0(0 \%)$ & $0(0 \%)$ & $0(0 \%)$ & 0.58 \\
Anal dermatitis & $2(7 \%)$ & $0(0 \%)$ & $0(0 \%)$ & $0(0 \%)$ & 0.29 \\
Anorectal infection & $0(0 \%)$ & $0(0 \%)$ & $0(0 \%)$ & $1(3 \%)$ & 0.56 \\
\hline
\end{tabular}

hyponatremia, which did not allow the patient to continue CRT. On the other hand, only 1 patient had Grade 3 anorectal infection in the UFT group. The incidence of Grade 3 diarrhea occurred with significant frequency in the $\mathrm{S}-1$ group compared with the UFT group $(\mathrm{p}=0.02)$.

The comparison of compliance of CRT is shown in Table 5. Twenty-five patients (83\%) received the scheduled CRT dose in the S-1 group. Three patients did not receive the planned total dose of S-1 by incidence of hematologic toxicity. One patient did not receive the planned total dose of radiation by incidence of Grade 2 anal dermatitis and received a total dose of $34 \mathrm{~Gy}$. One patient could not continue CRT. This patient had Grade 3 diarrhea/fatigue/hyponatremia and received a total dose of 30 Gy. Complete therapy of CRT was performed in 28 patients $(97 \%)$ in the UFT group. Only 1 patient did not receive the planned total dose of UFT by incidence of anorectal infection. 
Table 5. Comparison of compliance of CRT.

\begin{tabular}{cccc}
\hline & S-1 $(\mathrm{n}=30)$ & UFT $(\mathrm{n}=29)$ & $\mathrm{p}$ value \\
\hline Complete therapy & & & 0.21 \\
Yes & $25(83 \%)$ & $28(97 \%)$ & \\
No & $5(17 \%)$ & $1(3 \%)$ & \\
Discontinuance & & & \\
Only chemotherapy & $3(10 \%)$ & $1(3 \%)$ & 0.63 \\
Only radiotherapy & $1(3 \%)$ & $0(0 \%)$ & 0.99 \\
Chemoradiotherapy & $1(3 \%)$ & $0(0 \%)$ & 0.99 \\
\hline
\end{tabular}

\section{Discussion}

For the method of 5-FU administration, oral fluoropyrimidine is an attractive alternative to protracted infusion 5-FU without the need for intravenous access and infusion pumps [15]. Because the standard schedule of preoperative CRT for rectal cancer remains to be established, and due to the convenience of oral prodrugs of 5-FU, we evaluated the efficacy and toxicity of UFT or S-1 and preoperative radiotherapy in patients with locally advanced rectal cancer. Phase II studies of preoperative CRT in rectal cancer are commonly aimed to address pathological CR rate as a surrogate endpoint of efficacy because it closely correlates well with survival [16]. Hence, new strategies in the preoperative treatment of locally advanced rectal cancer should endeavor to attain satisfactory pathological CR rates, but improvement must outweigh any increases in toxicity associated with these new approaches.

Several phase II trials using UFT as a single agent, without LV modulation, concurrently with radiotherapy have been published. Vestermark et al. [9] treated 52 patients with either primary or recurrent rectal cancer with UFT $\left(300 \mathrm{mg} / \mathrm{m}^{2} /\right.$ day $)$ and l-LV $(22.5 \mathrm{mg}), 5$ days a week for 6 weeks and high-dose (60 Gy) pelvic radiotherapy including a concurrent boost, with a pathological CR rate of $13 \%$ for those patients undergoing an operation. Fernandez-Martos et al. [10] treated 94 patients with UFT ( $400 \mathrm{mg} / \mathrm{m}^{2} /$ day, 5 days a week for 5 weeks) and conventional-dose (45 Gy) pelvic radiotherapy. The pathological CR rate achieved in this study was a little lower $(9 \%$ pathological $\mathrm{CR})$, although $23 \%$ of patients had only residual microscopic foci and 54\% of patients were downstaged. De la Torre et al. [11] have recently published the results of a randomized phase III trial comparing 5FU/LV versus UFT/LV given concurrently with preoperative irradiation (45 - 50.4 Gy) in patients with locally advanced rectal cancer. The authors reported a pathological CR rate of $13 \%$ in both arms. However, tumor downstaging was more common with preoperative UFT/LV than with $5 \mathrm{FU} / \mathrm{LV}(59.2 \%$ versus $43.3 \%$; $\mathrm{p}=$ 0.04). In the report of Feliu et al. [8], $43 \%$ of patients receiving UFT $350 \mathrm{mg} / \mathrm{m}^{2}$ suffered Grade 3 - 4 gastrointestinal toxicity.

Other oral fluoropyrimidine derivatives also have been integrated in this preoperative CRT approach. Capecitabine in combination with radiotherapy has been evaluated as a preoperative treatment in phase II trials with a reported pathological CR rate of $12 \%$ - 24\% [17-19]. The combination of Capecitabine and radiotherapy in the preoperative setting also has a favorable safety profile. Grade 3 non-hematologic toxicity mainly consisted of diarrhea and proctitis, and Grade 3 leucopenia was seldom reported. Regarding S-1, our study is the first report using S-1 as single agent for CRT of locally advanced rectal cancer. Furthermore, we investigated the efficacy and toxicity of CRT using UFT and S-1 defined as oral DPD-inhibitory fluoropyrimidine. The results of this study clearly show that CRT of the total dose of 40 Gy with oral fluoropyrimidines in preoperative setting is well tolerated and effective in patients with locally advanced rectal cancer. In this study, the UFT dose was lower (300 $\mathrm{mg} / \mathrm{m}^{2} /$ day). While we observed only $7 \%$ Grade 3 diarrhea in the S-1 group, we achieved 7\% pathological CR and $47 \%$ downstaging in the S- 1 group, and $4 \%$ and $59 \%$ in the UFT group. Furthermore, we achieved $57 \%$ pathological response in the S-1 group and $45 \%$ in the UFT group.

Recently, preliminary reports from two randomized trials, STAR-01 [20] and ACCORD 12/0405 PRODIGE 1 [21], demonstrated that oxaliplatin added to preoperative CRT did not improve tumor response and significantly increased serious diarrhea. In this regard, there is legitimate concern that the combination of two drugs in preoperative CRT is not always sufficient to control locoregional lesions and eradicate distant metastases over fluoropyrimidine. On the other hand, Sato et al. [22] reported about a phase II trial of preoperative CRT with $\mathrm{S}-1$ plus irinotecan and showed that this regimen had high pathological CR rate of $37.3 \%$.

Glimelius et al. [23] explored the relationship between radiation dose alone and reduction in the local recurrence rate reported in randomized clinical trials. Wiltshire et al. conducted a radiation dose escalation study with $5 \mathrm{FU}$ $\left(225 \mathrm{mg} / \mathrm{m}^{2} /\right.$ day) given continuously throughout radiotherapy with 3 dose levels of 40, 46 and 50 Gy given at 2 Gy per fraction [24]. The authors reported a pathological CR of $15 \%, 23 \%$ and $33 \%$, respectively. According to the univariate analysis, the only factor found to be predictive of locoregional control, disease free survival, and overall survival was the dose. This increase in radiotherapy dose was not associated with an increase in Grade 3 4 acute gastrointestinal toxicity. Mohiuddin et al. [25] found that patients treated up to a dose of $<50 \mathrm{~Gy}$, even in the presence of chemotherapy, had a pathological CR 
rate of $3 \%$, compared with a pathological CR rate of $45 \%$ at doses of $>55$ Gy $(p<0.05)$. In this study, although the pathological CR rate was similar to those studies using a lower radiation dose, satisfactory pathological $\mathrm{CR}$ rate may need a higher radiation dose.

As CDHP shows a 180 times higher DPD-inhibitory effect compared with uracil and has potent radiosensitizing property, we thought that $\mathrm{S}-1$ might become an active drug for CRT of locally advanced rectal cancer. However, there was no statistically significant difference between the S-1 group and the UFT group in regard to the pathological response rate and pathological CR rate. For this reason, we think that small sample in this study may have influenced the similarity of pathological response rate and pathological CR rate. Additionally, in S-1, Oxo is also combined to prevent GI toxicity without loss of the antitumor effect, as Oxo inhibits phosphorylation of 5-FU in the GI tract but not in tumor tissues. This may lead to the outcome that the main toxicity in the $\mathrm{S}-1$ group was only $7 \%$ Grade 3 diarrhea in spite of its high antitumor effect.

The key weaknesses of this report are as follows: no double-blind randomized study, small sample, possibility including anal cancer and no data including surgical complication, local control, overall and disease free survival. However, CRT with oral fluoropyrimidines in preoperative setting showed satisfactory downstaging and pathological response rate in the both group. These results suggest that CRT with oral fluoropyrimidines in this study is effective and feasible. It will be necessary to confirm the usefulness of CRT with oral fluoropyrimidines by larger prospective studies in the future.

\section{Conclusion}

Although long-term oncologic results require further investigation, preoperative CRT using UFT or S-1 is effective and feasible for patients with locally advanced rectal cancer.

\section{Acknowledgements}

Grant support was provided by the Research Support Foundation of The University of Tokushima and TAIHO Pharmaceutical Co., LTD., and the Grants-in-Aid for Scientific Research of the Japan Society for the Promotion of Science. A part of this study was presented in Gastrointestinal Cancer Symposium, San Francisco, CA, USA (Jan. 20-22, 2011).

\section{REFERENCES}

[1] C. Camma, M. Giunta, F. Fiorica, L. Pagliaro, A. Craxi and M. Cottone, "Preoperative Radiotherapy for Re- sectable Rectal Cancer: A Meta-Analysis," Journal of the American Medical Association, Vol. 284, No. 8, 2000, pp. 1008-1015. doi:10.1001/jama.284.8.1008

[2] Colorectal Cancer Collaborative Group, “Adjuvant Radiotherapy for Rectal Cancer: A Systematic Overview of 8,507 Patients from 22 Randomised Trials," Lancet, Vol. 358, No. 9290, 2001, pp. 1291-1304. doi:10.1016/S0140-6736(01)06409-1

[3] J. F. Bosset, L. Collette, G. Calais, L. Mineur, P. Maingon, L. Radosevic-Jelic, A. Daban, E. Bardet, A. Beny and J. C. Ollier, EORTC Radiotherapy Group Trial 22921, "Chemotherapy with Preoperative Radiotherapy in Rectal Cancer," The New England Journal of Medicine, Vol. 355, 2006, pp. 1114-1123. doi:10.1056/NEJMoa060829

[4] S. Fujii, S. Kitano, K. Ikenaka and T. Shirasaka, "Effect of coadministration of Uracil Or Cytosine on the AntiTumor Activity of Clinical Doses of 1-(2-Tetrahydrofuryl)-5-Fluorouraciland Level of 5-Fluorouracil in Rodents," Japanese Journal of Cancer Research: Gann, Vol. 70, No. 2, 1979, pp. 209-214.

[5] T. Shirasaka, Y. Shimamato, H. Ohshimo, M. Yamaguchi, T. Kato, K. Yonekura and M. Fukushima, "Development of A Novel Form of an Oral 5-Fluorouracil Derivative (S-1) Directed to the Potentiation of the Tumor Selective Cytotoxicity of 5-Fluorouracil by Two Biochemical Modulators," Anti-Cancer Drugs, Vol. 7, No. 5, 1996, pp. 548-557. doi:10.1097/00001813-199607000-00010

[6] T. Shirasaka, K. Nakano, T. Takechi, H. Satake, J. Uchida, A. Fujioka, H. Saito, H. Okabe, K. Oyama, S. Takeda, N. Unemi and M. Fukushima, "Antitumor Activity of $1 \mathrm{M}$ Tegafur-0.4 M 5-Chloro-2,4-Dihydroxypyridine-1 M Potassium Oxonate (S-1) against Human Colon Carcinoma Orthotopically Implanted into Nude Rats," Cancer Research, Vol. 56, No. 11, 1996, pp. 2602-2606.

[7] P. M. Hoff, N. Janjan, E. D. Saad, J. Skibber, C. Crane, Y. Lassere, K. R. Cleary, S. Benner, J. Randolph, J. L. Abbruzzese and R. Pazdur, "Phase I Study of Preoperative Oral Uracil and Tegafur plus Leucovorin and Radiation Therapy in Rectal Cancer," Journal of Clinical Oncology, Vol. 18, No. 20, 2000, pp. 3529-3534.

[8] J. Feliu, J. Calvilio, A. Escribano, J. de Castro, M. E. Sanchez, A. Mata, E. Espinosa, A. G. Grande, A. Mateo and M. G. Baron, "Neoadjuvant Therapy of Rectal Carcinoma with Uft-Leucovorin plus Radiotherapy," Annals of Oncology, Vol. 13, No. 5, 2002, pp. 730-736. doi:10.1093/annonc/mdf116

[9] L. W. Vestermark, A. Jacobsen, C. Qvortrup, F. Hansen C. Bisgaard, G. Baatrup, P. Rasmussen and P. Pfeiffer, "Long-Term Results of a Phase II Trial of High Dose Radiotherapy (60 Gy) and UFT/1-Leucovorin in Patients with Non-Resectable Locally Advanced Rectal Cancer (LARC)," Acta Oncologica, Vol. 47, No. 3, 2008, pp. 428-433. doi:10.1080/02841860701798866

[10] C. Fernández-Martos, J. Aparicio, C. Bosch, M. Torregrosa, J. M. Campos, S. Garcera, J. M. Vicent, I. Maestu, M. A. Climent, J. L. Mengual, A. Tormo, A. Hernandez, R. Estevan J. M. Richart, V. Viciano, N. Uribe, J. Campos, R. Puchades, F. Arlandis and D. Al- 
menar, "Preoperative Uracil, Tegafur, and Concomitant Radiotherapy in Operable Rectal Cancer: A Phase II Multicenter Study with 3 Years' Follow-Up," Journal of Clinical Oncology, Vol. 22, No. 15, 2004, pp. 3016-3022. doi:10.1200/JCO.2004.11.124

[11] A. de la Torre, M. I. García-Berrocal, F. Arias, A. Mariño, F. Valcárcel, R. Magallón, C. A. Regueiro, J. Romero, I. Zapata, C. de la Fuente, E. Fernandez-Lizarbe, G. Vergara, B. Belinchon, M. Veiras, R. Moleron and I. Millan, "Preoperative Chemoradiotherapy for Rectal Cancer: Randomized Trial Comparing Oral Uracil and Tegafur and Oral Leucovorin vs. Intravenous 5-Fluorouracil and Leucovorin," International Journal of Radiation Oncology, Biology, Physics, Vol. 70, No. 1, 2008, pp. 102-110. doi:10.1016/j.ijrobp.2007.05.068

[12] M. Fukushima, K. Sakamoto, M. Sakata, F. Nakagawa, H. Saito and Y. Sakata, "Gimeracil, a Component of S-1, May Enhance the Antitumor Activity of X-Ray Irradiation in Human Cancer Xenograft Models in Vivo," Oncology Reports, Vol. 24, No. 5, 2010, pp. 1307-1313. doi:10.3892/or 00000987

[13] S. Morimoto, M. Shimada, N. Kurita, H. Sato, T. Iwata, M. Nishioka, K. Yoshikawa, T. Miyatani, H. Kashihara, C. Takasu and H. Ikushima, "Preoperative Radiotherapy Combined with S-1 for Advanced Lower Rectal Cancer: Phase I Trial," Hepatogastroenterology, Vol. 59, No. 117, 2012, pp. 1428-1432. doi:10.5754/hge11699

[14] "Response Assessment of Nonsurgical Treatment for Colorectal Carcinoma. Japanese Classification of Colorectal Carcinoma. Japanese Society for Cancer of the Colon and Rectum," 1st English Edition, Kanehara \& Co. Ltd., Tokyo, 1997, pp. 77-82.

[15] R. Glynne-Jones, J. Dunst and D. Sebag-Montefiore, "The Integration of Oral Capecitabine into Chemoradiation Regimens for Locally Advanced Rectal Cancer: How Successful Have We Been?" Annals of Oncology, Vol. 17, No. 3, 2006, pp. 361-371. doi:10.1093/annonc/mdj052

[16] N. A. Janjan, J. Abbruzzese, R. Pazdur, V. S. Khoo, K. Cleary, R. Dubrow, J. Ajani, T. A. Rich, M. S. Goswitz, P. A. Evetts, P. K. Allen, P. M. Lynch and J. M. Skibber, "Prognostic Implications of Response to Preoperative Infusional Chemoradiation in Locally Advanced Rectal Cancer," Radiotherapy \& Oncology, Vol. 51, No. 2, 1999, pp. 153-160. doi:10.1016/S0167-8140(99)00054-7

[17] J. Dunst, T. Reese, T. Sutter, H. Zühlke, A. Hinke, K. Kölling-Schlebusch and S. Frings, "Phase I Trial Evaluating the Concurrent Combination of Radiotherapy and Capecitabine in Rectal Cancer," Journal of Clinical Oncology, Vol. 20, No. 19, 2002, pp. 3983-3991. doi:10.1200/JCO.2002.02.049

[18] J. Souglakos, N. Androulakis, D. Mavroudis, C. Kourousis, S. Kakolyris, N. Vardakis, K. Kalbakis, A. Pallis, A. Ardavanis, C. Varveris and V. Georgoulias, "Multicenter Dose-Finding Study of Concurrent Capecitabine and Radiotherapy as Adjuvant Treatment for Operable Rectal Cancer," International Journal of Radiation Oncology, Biology, Physics, Vol. 56, No. 5, 2003, pp. 1284-

\section{7. doi:10.1016/S0360-3016(03)00275-X}

[19] I. Craven, A. Crellin, R. Cooper, A. Melcher, P. Byrne and D. Sebag-Montefiore, "Preoperative Radiotherapy Combined with 5 Days Per Week Capecitabine Chemotherapy in Locally Advanced Rectal Cancer," British Journal of Cancer, Vol. 97, No. 10, 2007, pp. 1333-1337. doi:10.1038/sj.bjc. 6604042

[20] C. Aschele, C. Pinto, S. Cordio, G. Rosati, A. Tagliagambe, S. Artale, P. Rosetti, S. Lonardi, L. Boni and L. Cionini, "Preoperative Fluorouracil (FU)-Based Chemoradiation with and Without Weekly Oxaliplatin in Locally Advanced Rectal Cancer: Pathologic Response Analysis of the Studio Terapia Adiuvante Reto (STAR)-01 Randomized Phase III Trial (Abstract \#4008)," Journal of Clinical Oncology, Vol. 27, 2009, p. 804.

[21] J. Gerard, D. Azria, S. Gourgou-Bourgade, I. Martel-Laffay, C. Hennequin, P. L. Etienne, V. Vendrely, E. Francois, G. de La Roche, O. Bouche, X. Mirabel, B. Denis, L. Mineur, J. F. Berdah, M. A. Mahe, Y. Becouarn, O. Dupuis, G. Lledo, C. Montoto-Grillot and T. Conroy, "Comparison of Two Neoadjuvant Chemoradiotherapy Regimens for Locally Advanced Rectal Cancer: Results of the Phase III Trial ACCORD 12/0405-Prodige 2," Journal of Clinical Oncology, Vol. 28, No. 10, 2010, pp. 1638-1644. doi:10.1200/JCO.2009.25.8376

[22] T. Sato, H. Ozawa, K. Hatate, W. Onosato, M. Naito, T. Nakamura, A. Ihara, W. Koizumi, K. Hayakawa, I. Okayasu, K. Yamashita and M. Watanabe, "A Phase II Trial of Neoadjuvant Preoperative Chemoradiotherapy with S-1 plus Irinotecan and Radiation in Patients with Locally Advanced Rectal Cancer: Clinical Feasibility and Response Rate," International Journal of Radiation Oncology, Biology, Physics, Vol. 79, No. 3, 2011, pp. 677-683. doi:10.1016/j.ijrobp.2009.11.007

[23] B. Glimelius, U. Isacsson, B. Jung and L. Pahlman, "Radiotherapy in Addition to Radical Surgery in Rectal Cancer: Evidence for a Dose-Response Effect Favoring Preoperative Treatment," International Journal of Radiation Oncology, Biology, Physics, Vol. 37, No. 2, 1997, pp. 281-287. doi:10.1016/S0360-3016(96)00510-X

[24] K. L. Wiltshire, I. G. Ward, C. Swallow, A. M. Oza, B. Cummings, G. R. Pond, P. Catton, J. Kim, J. Ringash, C. S. Wong, R. Wong, L. L. Siu, M. Moore and J. Brierley, "Preoperative Radiation with Concurrent Chemotherapy for Resectable Rectal Cancer: Effect of Dose Escalation on Pathologic Complete Response, Local RecurrenceFree Survival, Disease-Free Survival, and Overall Survival," International Journal of Radiation Oncology, Biology, Physics, Vol. 64, No. 3, 2006, pp. 709-716. doi:10.1016/j.ijrobp.2005.08.012

[25] M. Mohiuddin, W. F. Regine, W. J. John, P. F. Hagihara, P. C. McGrath, D. E. Kenady and G. Marks, "Preoperative Chemoradiation in Fixed Distal Rectal Cancer: Dose Time Factors for Pathological Complete Response," International Journal of Radiation Oncology, Biology, Physics, Vol. 46, No. 4, 2000, pp. 883-888. doi:10.1016/S0360-3016(99)00486-1 